\title{
Iterative Soft Interference Cancellation Aided Minimum Bit Error Rate Uplink Receiver Beamforming
}

\author{
S. Tan, L. Xu, S. Chen, and L. Hanzo \\ School of Electronics and Computer Science \\ University of Southampton, Southampton, SO17 1BJ, U.K. \\ $\{$ st104r,lx04r,sqc,lh\}@ecs.soton.ac.uk
}

\begin{abstract}
Iterative multiuser receivers constitute an effective solution for transmission over Multiple Access Interference (MAI) infested channels, when invoking a combined multiuser detector and channel decoder. Most reduced-complexity methods in this area use the Complex-valued Minimum Mean Squared Error (CMMSE) Multiuser Detector (MUD). Since the desired output of BPSK systems is real-valued, minimizing the Mean Square Error (MSE) between the beamformer's desired output and the real part of the beamformer output has the potential of significantly improving the attainable Bit Error Rate (BER) performance. We refer to this MMSE design as the Real-valued MMSE (RMMSE) receiver. In this paper, we explore a new Soft-Input Soft-Output (SISO) interference cancellation multiuser detection algorithm based on the novel Minimum BER (MBER) criterion. We demonstrate that the MBER turbo receiver outperforms both the CMMSE and the RMMSE algorithms, particularly in so-called 'overloaded' beamforming systems, where the number of receiver antennas is lower than the number of users supported.
\end{abstract}

\section{INTRODUCTION}

The increasing demand for mobile communication services supported within a limited radio-frequency bandwidth motivates the design of antenna array assisted beamforming techniques [1] as well as Spatial Division Multiple Access (SDMA) arrangements [2]. By appropriately combining the signals received by the different elements of an antenna array, beamforming becomes capable of creating an angularly selective transmitter/receiver beam, hence potentially separating signals transmitted on the same carrier frequency but arriving from sufficiently different angles.

Since the discovery of turbo codes [3], iterative detection [4] has been applied in the context of joint channel estimation and equalization [5], in multiuser detection [6] and numerous other coded communication systems [7]-[9]. In iterative multiuser receivers, the MUD and the channel decoder exchange extrinsic information in a number of consecutive iterations. During each iteration, the extrinsic information extracted from both the MUD and the channel decoder is used as the a priori input by the other stage in the next iteration. The information exchanged is exploited for the sake of improving the receiver's attainable performance. In [7], a suboptimal linear MUD was introduced, which benefitted from both soft interference cancellation and instantaneous linear minimum mean squared error filtering.

Against this background, in this contribution we propose a novel iterative beamforming receiver. The conventional beamformer combines the signals received with the aid of each antenna element for the sake of minimizing the MSE between the complex-valued locally stored and received reference signal. We will refer to this MMSE solution as the Complex-valued MMSE (CMMSE) scheme.

The insightful suggestions of Volker Kühn are gratefully acknowledged. Furthermore, the financial support of the EPSRC, UK and the European Union in the framework of the Phoenix as well as Newcom projects is thankfully acknowledged by the authors.
For BPSK systems, however, the beamformer's desired output is realvalued. By minimizing the MSE between the beamformer's desired output and the real part of the beamformer output, the achievable system's BER performance can significantly be enhanced [10]. We will refer to this alternative MMSE design as the Real-valued MMSE (RMMSE) arrangement in order to contrast it with the standard CMMSE. However, the CMMSE and RMMSE algorithms do not guarantee the direct and explicit minimization of the system's BER, despite the monotonous relationship of the MSE and the BER. Hence in references [11]-[13] the BER rather/local/st104rthan the MSE was minimized at the MUD's output. The Minimum BER (MBER) beamforming design is the true optimal solution and hence it generally outperforms the CMMSE and the RMMSE solutions, particularly in the context of the so-called 'overloaded' systems, where the degree of freedom of the antenna array is lower than the number of users. The achievable BER difference of the MMSE and MBER receivers becomes particularly dramatic in this scenario.

The structure of this contribution is as follows. In Section II, we outline the signal model used, followed by the portrayal of our iterative beamformer design. The focus of Section III is the SoftIn Soft-Out (SISO) interference canceller advocated. Our simulation results are presented in Section IV, followed by our conclusions in Section V.

\section{SYSTEM DESCRIPTION}

\section{A. Signal Model}

The system supports $K$ users, and each user transmits his/her signal on the same carrier frequency of $\omega=2 \pi f$. The receiver is equipped with a linear antenna array consisting of $L$ elements, which have a uniform element spacing of $\lambda / 2$, as shown in Fig. 1, where $\lambda$ is the wavelength. Assume that the channel is non-dispersive in both the angular and the time domain and hence does not induce Intersymbol Interference (ISI). Then the symbol-rate received signal samples can be expressed as

$$
r_{l}(n)=\sum_{k=1}^{K} A_{k} s_{k}(n) e^{j \omega t_{l}\left(\theta_{k}\right)}+n_{l}(n)
$$

for $1 \leq l \leq L$, where $A_{k}$ is the non-dispersive complex-valued channel coefficient of user $k, s_{k}(n)$ is the $n$th symbol of user $k, n_{l}(n)$ is a complex-valued Gaussian white noise process associated with $E\left[\left|n_{l}(n)\right|^{2}\right]=2 \sigma_{n}^{2}$, and

$$
t_{l}\left(\theta_{k}\right)=\frac{\lambda}{2}(l-1) \sin \left(\theta_{k}\right) / c
$$

is the relative time delay at array element $l$ for the source signal of user $k$, with $\theta_{k}$ being the Line Of Sight (LOS) angle of arrival for source $k$, and $c$ is the speed of light. 


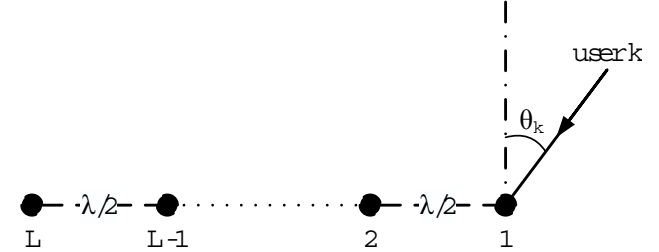

Fig. 1. Geometric structure of the antenna array showing the received signal of user $k$, where $\theta_{k}$ is the LOS component's angle of arrival

If source $k$ is the desired user and the rest of the sources are the interfering users, then the desired-user's Signal to Noise Ratio becomes $\mathrm{SNR}_{k}=\left|A_{k}\right|^{2} \sigma_{s}^{2} / 2 \sigma_{n}^{2}$ and the deschoberrsired Signal to Interference Ratio of user $k^{\prime}$ is $\operatorname{SIR}_{k, k^{\prime}}=\left|A_{k}\right|^{2} /\left|A_{k^{\prime}}\right|^{2}$, where $\sigma_{s}^{2}=1$ is the symbol energy. The received signal vector $\mathbf{r}(n)=$ $\left[r_{1}(n) r_{2}(n) \cdots r_{L}(n)\right]^{T}$ is given by

$$
\mathbf{r}(n)=\mathbf{H} \mathbf{s}(n)+\mathbf{n}(n),
$$

where we have $\mathbf{n}(n)=\left[n_{1}(n) n_{2}(n) \cdots n_{L}(n)\right]^{T}$, the transmitted symbol vector of the $K$ users is $\mathbf{s}(n)=$ $\left[s_{1}(n) s_{2}(n) \cdots s_{K}(n)\right]^{T}$, and the system matrix is denoted by $\mathbf{H}=\left[\mathbf{h}_{1} \mathbf{h}_{2} \cdots \mathbf{h}_{K}\right]$ which is associated with the steering vector

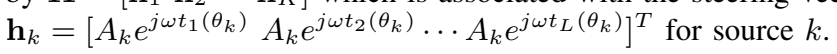

\section{B. Iterative Multiuser Beamforming Receiver Structure}

The iterative multiuser beamforming receiver's structure is shown in Fig. 2, which consists of two stages, namely the SISO interference cancellation aided beamforming multiuser detector, followed by $K$ parallel single-user SISO channel decoders. The two stages are separated by the usual deinterleavers and interleavers.

The proposed SISO beamforming MUD first computes the estimated symbol $\hat{s}_{k}$ corresponding to the transmitted symbol $s_{k}$ using a linear filter, which determines the coefficients of the beamformer weight $\mathbf{w}$ according to the specific design criterion employed and uses this weight to estimate $\hat{s}_{k}$ from the received signal $\mathbf{r}$ with the aid of a linear transformation [6]. Let us now define $b_{k}(n, i)$ as the $i$ th bit of symbol $s_{k}(n)$, whereas $b_{k}(j)$ is the same bit but in a different position of the bit-based interleaving block after the deinterleaver. The indices $m$ and $c$ are associated with the multiuser detector and channel decoder, respectively, and the indices $p r, p o$ and $e$ are used for the a priori, a posteriori and extrinsic information. Then the SISO beamforming MUD delivers the a posteriori information of bit $b_{k}(n, i)$ expressed in terms of its Log-Likelihood Ratio (LLR) as [4]

$$
\begin{aligned}
L_{m, p o, b_{k}(n, i)} & =\ln \frac{\mathrm{P}\left[b_{k}(n, i)=0 \mid \hat{s}_{k}(n)\right]}{\mathrm{P}\left[b_{k}(n, i)=1 \mid \hat{s}_{k}(n)\right]} \\
& =\ln \frac{\mathrm{P}\left[\hat{s}_{k}(n) \mid b_{k}(n, i)=0\right]}{\mathrm{P}\left[\hat{s}_{k}(n) \mid b_{k}(n, i)=1\right]}+\ln \frac{\mathrm{P}\left[b_{k}(n, i)=0\right]}{\mathrm{P}\left[b_{k}(n, i)=1\right]} \\
& =L_{m, e, b_{k}(n, i)+L_{m, p r, b_{k}(n, i)},}
\end{aligned}
$$

where the second term, denoted by $L_{m, p r, b_{k}(n, i)}$, represents the $a$ priori LLR of the interleaved and Recursive Systematic Convolutional (RSC) encoded bit $b_{k}(n, i)$. For the first iteration, assuming equiprobable RSC encoded bits, i.e. that no a priori information is available, all bits have a probability of 0.5 . Hence in the LLR domain we have $L_{m, p r, b_{k}(n, i)}=0$. The first term in Equation (4), which is denoted by $L_{m, e, b_{k}(n, i)}$, represents the extrinsic information delivered by the SISO multiuser detector, based on the received signal $\mathbf{r}(n)$ and on the $a$ priori information about the RSC encoded bits of all users, except the $i$ th bit of the desired user $k$. The extrinsic information, which is not influenced by the a priori information of the desired bit $i$ provided by the $k$ th channel decoder, is then deinterleaved and fed into the $k$ th user's channel decoder, which will be used as the $a$ priori information in the next iteration.

As seen in Fig. 2, between the banks of channel decoders and interleavers, based on the a priori information $L_{c, p r, b_{k}(j)}$ provided by the SISO beamforming MUD for the SISO decoder, we compute the extrinsic LLR as [4]

$$
L_{c, e, b_{k}(j)}=L_{c, p o, b_{k}(j)}-L_{c, p r, b_{k}(j)},
$$

where the extrinsic information is gleaned from the surrounding RSC encoded bits, excluding the specific bit considered [5]. We note that as usual in joint iterative detection and decoding schemes [5], we exchange the extrinsic information concerning both the original information bits and parity bits, rather than only that of the information bits, although only the LLRs of the latter are needed in the classic turbo decoder of Berrou et al. [3]. After interleaving, the extrinsic information delivered by the channel decoders is then fed back to the SISO multiuser detector, as the a priori information concerning the RSC encoded bits of all the users for exploitation during the next iteration.

At the first iteration, the extrinsic information contributions $L_{m, e, b_{k}}$ and $L_{c, e, b_{k}}$ are statistically independent. However, during the subsequent iterations they will become more and more correlated and hence the incremental iteration gains become more modest.

\section{SISO INTERFERENCE CANCELLATION}

As described in the previous section, the task of SISO interference cancellation is to choose the beamformer weight $\mathbf{w}$ of the linear filter seen in Fig. 2 according to an appropriate design criterion and compute the corresponding output LLRs.

Given the a priori LLRs, we first define the mean and covariance of the $k$ th user's symbols as [8]

$$
\begin{aligned}
\bar{s}_{k}=\mathrm{E}\left[s_{k}\right]=\sum_{q} s_{k}^{(q)} \cdot \mathrm{P}\left(s_{k}=s_{k}^{(q)}\right) \\
= \begin{cases}\tanh \left(\frac{L_{p r, b_{k}(1)}}{2}\right), & \text { BPSK } \\
\frac{\tanh \left(\frac{L_{p r, b_{k}(1)}}{2}\right)+j \tanh \left(\frac{L_{p r, b_{k}(2)}}{2}\right)}{\sqrt{2}}, & \text { QPSK }\end{cases}
\end{aligned}
$$

and

$$
\begin{aligned}
v_{k} & =\operatorname{Cov}\left[s_{k}, s_{k}\right]=\mathrm{E}\left[s_{k} s_{k}^{*}\right]-\mathrm{E}\left[s_{k}\right] \mathrm{E}\left[s_{k}^{*}\right] \\
& = \begin{cases}1-\tanh ^{2}\left(\frac{L_{p r, b_{k}(1)}}{2}\right), & \text { BPSK } \\
1-\frac{\tanh ^{2}\left(\frac{L_{p r, b_{k}(1)}}{2}\right)+\tanh ^{2}\left(\frac{L_{p r, b_{k}(2)}}{2}\right)}{2}, & \text { QPSK , }\end{cases}
\end{aligned}
$$

where $s_{k}^{(q)}$ is the $q$ th legitimate value of the symbol $s_{k}$. When using the soft interference cancellation principle, the estimated symbol of user $k$ can be expressed as [8]

$$
\hat{s}_{k}=\bar{s}_{k}+v_{k} \mathbf{w}_{k}^{H}(\mathbf{r}-\mathbf{H} \overline{\mathbf{s}}),
$$

where we have $\overline{\mathbf{s}}=\left[\begin{array}{lll}\bar{s}_{1} & \bar{s}_{2} \cdots \bar{s}_{K}\end{array}\right]^{T}$.

A. SISO Interference Cancellation Using the Complex-Valued MMSE MUD

Classically, the beamformer's weight vector $\mathbf{w}_{k}$ is determined by minimizing the complex-valued MSE metric of [1]

$$
J_{c m s e}\left(\mathbf{w}_{k}\right)=\mathrm{E}\left[\left|s_{k}-\hat{s}_{k}\right|^{2}\right] .
$$




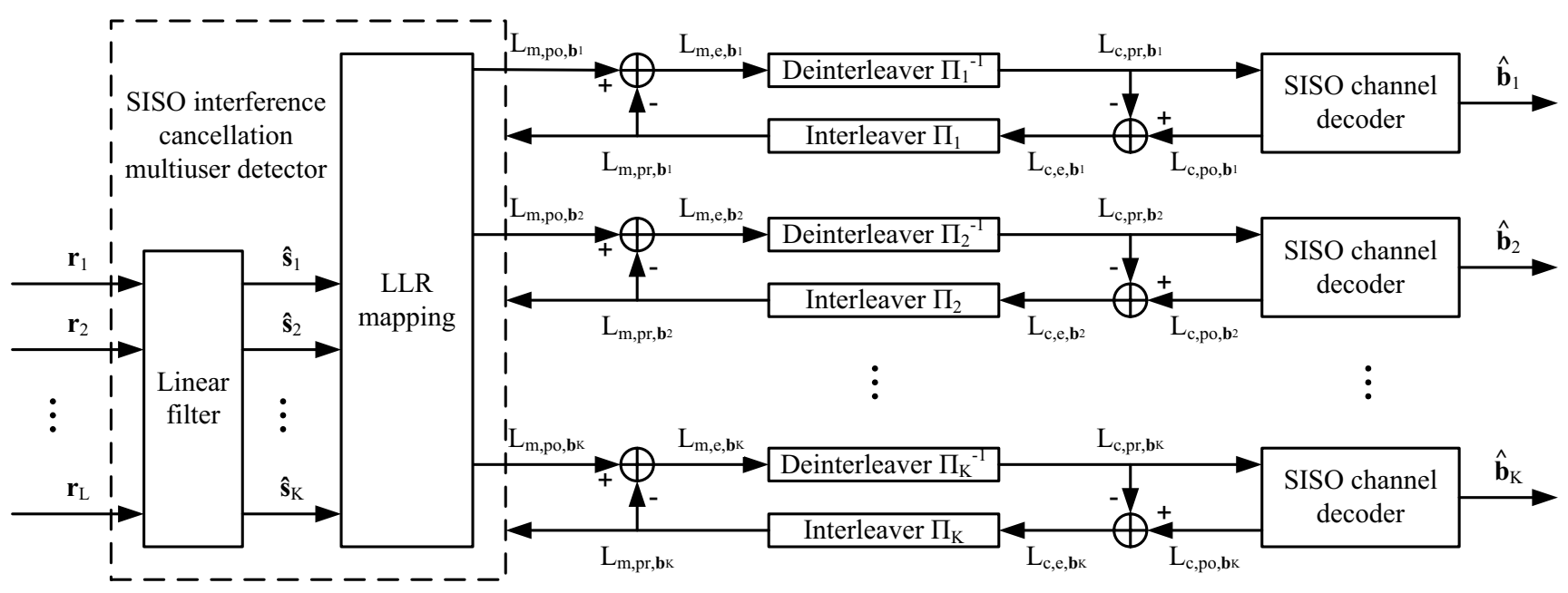

Fig. 2. Iterative multiuser beamforming receiver structure

Using Equation (8) and setting the gradient of $J_{c m s e}\left(\mathbf{w}_{k}\right)$ in Equation (9) to zero leads to the closed-form CMMSE solution of [8]

$$
\mathbf{w}_{k, c m m s e}=\left(\mathbf{H V H} \mathbf{H}^{H}+2 \sigma_{n}^{2} \mathbf{I}_{L}\right)^{-1} \mathbf{h}_{k},
$$

where $\mathbf{I}_{L}$ denotes the $(L \times L)$-dimensional identity matrix and we have $\mathbf{V}=\operatorname{diag}\left[v_{1} v_{2} \cdots v_{K}\right]$.

As stated in [8], the conditional PDF $\mathrm{P}\left[\hat{s}_{k} \mid s_{k}=s_{k}^{(q)}\right]$ can be assumed to be Gaussian distributed and the extrinsic output LLRs are given by

$$
L_{e, b_{k}(1)}=\frac{4 \Re\left[\mathbf{w}_{k}^{H}\left(\mathbf{r}-\mathbf{H} \overline{\mathbf{s}}+\bar{s}_{k} \mathbf{h}_{k}\right)\right]}{1-v_{k} \mathbf{w}_{k}^{H} \mathbf{h}_{k}}
$$

for BPSK, and

$$
\begin{aligned}
L_{e, b_{k}(1)} & =\frac{2 \sqrt{2} \Re\left[\mathbf{w}_{k}^{H}\left(\mathbf{r}-\mathbf{H} \overline{\mathbf{s}}+\bar{s}_{k} \mathbf{h}_{k}\right)\right]}{1-v_{k} \mathbf{w}_{k}^{H} \mathbf{h}_{k}} \\
L_{e, b_{k}(2)} & =\frac{2 \sqrt{2} \Im\left[\mathbf{w}_{k}^{H}\left(\mathbf{r}-\mathbf{H} \overline{\mathbf{s}}+\bar{s}_{k} \mathbf{h}_{k}\right)\right]}{1-v_{k} \mathbf{w}_{k}^{H} \mathbf{h}_{k}}
\end{aligned}
$$

for QPSK.

\section{B. SISO Interference Cancellation Using the Real-Valued MMSE $M U D$}

For BPSK systems, the beamformer's desired output $s_{k}$ is realvalued. It is clear that the CMMSE solution of Section III-A attempts to simultaneously minimize the MSE between the desired signal and both the real part and imaginary part of the beamformer's output. However, in case of BPSK modulation the beamformer's decision depends only on the real part. Minimizing the MSE associated with the imaginary part does not contribute to improving the beamformer's performance. Rather it imposes an unnecessary constraint on the beamforming weight [10]. Hence we introduce the RMMSE solution.

The real-valued MSE cost function minimizing the MSE between the desired signal and the real part of the beamformer's output can be written as

$$
J_{r m s e}\left(\mathbf{w}_{k}\right)=\mathrm{E}\left[\left(s_{k}-\hat{s}_{k, R}\right)^{2}\right]
$$

where $\hat{s}_{k, R}=\Re\left[\hat{s}_{k}\right]$. The RMMSE solution is defined by

$$
\mathbf{w}_{k, r m m s e}=\arg \min _{\mathbf{w}} J_{r m s e}\left(\mathbf{w}_{k}\right) .
$$

The gradient of $J_{r m s e}\left(\mathbf{w}_{k}\right)$ is

$$
\begin{aligned}
\nabla J_{r m s e}\left(\mathbf{w}_{k}\right)= & -2 v_{k}^{2} \mathbf{h}_{k}+v_{k}^{2}\left(\mathbf{H} \mathbf{V} \mathbf{H}^{H}+2 \sigma_{n}^{2} \mathbf{I}_{L}\right) \mathbf{w}_{k} \\
& +v_{k}^{2} \mathbf{H} \mathbf{V} \mathbf{H}^{T} \mathbf{w}_{k}^{*} .
\end{aligned}
$$

It is seen in Equation (16) that there exists no closed-form solution for this RMMSE design. Fortunately, we can apply the real-valued vertical concatenation matrix method [10] to resolve this problem. Let us define the index $c$ as the subscript to indicate the matrices' vertical concatenation, then we have

$$
\mathbf{M}_{c}=\left(\begin{array}{c}
\Re[\mathbf{M}] \\
\Im[\mathbf{M}]
\end{array}\right)
$$

where $\mathbf{M}_{c}$ can be any matrix which is vertically concatenated. Hence, the gradient of Equation (14) becomes

$$
\nabla J_{r m s e}\left(\mathbf{w}_{k, c}\right)=-2 v_{k}^{2} \mathbf{h}_{k, c}+2 v_{k}^{2}\left(\mathbf{H}_{c} \mathbf{V} \mathbf{H}_{c}^{T}+\sigma_{n}^{2} \mathbf{I}_{2 L}\right) \mathbf{w}_{k, c} .
$$

Then in contrast to the closed-form CMMSE solution of Equation (10), the closed-form solution of the concatenated weight matrix $\mathbf{w}_{k, r m m s e, c}$ is derived from Equation (18), yielding

$$
\mathbf{w}_{k, r m m s e, c}=\left(\mathbf{H}_{c} \mathbf{V H}_{c}^{T}+\sigma_{n}^{2} \mathbf{I}_{2 L}\right)^{-1} \mathbf{h}_{k, c} .
$$

The first $L$ elements of $\mathbf{w}_{k, r m m s e, c}$ are the real part of the RMMSE solution $\mathbf{w}_{k, r m m s e}$, and the last $L$ elements of $\mathbf{w}_{k, r m m s e, c}$ form the imaginary part of $\mathbf{w}_{k, r m m s e}$.

The conditional $\operatorname{PDF} \mathrm{P}\left(\hat{s}_{k} \mid s_{k}=s_{k}^{(q)}\right)$ is a mixture of all legitimate transmitted signals' distributions, when the $k$ th user transmits symbol $s_{k}^{(q)}$ and all other interfering users transmit an arbitrary symbol. Unlike in case of the CMMSE solution, this conditional PDF cannot be assumed to be Gaussian distributed in the RMMSE design. The MSE minimization of the RMMSE solution considers only the inphase component and we assume that the real part of the PDF is Gaussian [14]. The conditional mean and variance of $\hat{s}_{k, R}$ are given by

$$
\begin{aligned}
\mu_{k, R}^{(q)} & =\mathrm{E}\left[\hat{s}_{k, R} \mid s_{k}=s_{k}^{(q)}\right] \\
& =\bar{s}_{k}+v_{k}\left(s_{k}^{(q)}-\bar{s}_{k}\right) \Re\left[\mathbf{w}_{k}^{H} \mathbf{h}_{k}\right]
\end{aligned}
$$


and

$$
\begin{aligned}
\sigma_{k, R}^{2}= & \operatorname{Cov}\left[\hat{s}_{k, R}, \hat{s}_{k, R} \mid s_{k}=s_{k}^{(q)}\right] \\
= & \frac{1}{2} v_{k}^{2} \mathbf{w}_{k}^{H}\left(\mathbf{H} \mathbf{V} \mathbf{H}^{H}-v_{k} \mathbf{h}_{k} \mathbf{h}_{k}^{H}+2 \sigma_{n}^{2} \mathbf{I}_{L}\right) \mathbf{w}_{k} \\
& +\frac{1}{2} v_{k}^{2} \Re\left[\mathbf{w}_{k}^{H}\left(\mathbf{H} \mathbf{V} \mathbf{H}^{T}-v_{k} \mathbf{h}_{k} \mathbf{h}_{k}^{T}\right) \mathbf{w}_{k}^{*}\right] .
\end{aligned}
$$

Given

$$
\mathrm{P}\left(\hat{s}_{k, R} \mid \mathbf{r}, s_{k}=s_{k}^{(q)}\right)=\frac{1}{\sqrt{2 \pi} \sigma_{k, R}} \exp \left(-\frac{\left(\hat{s}_{k, R}-\mu_{k, R}^{(q)}\right)^{2}}{2 \sigma_{k, R}^{2}}\right),
$$

the extrinsic output LLRs can be expressed as

$$
\begin{aligned}
L_{e, b_{k}(1)} & =\ln \frac{\mathrm{P}\left(\hat{s}_{k, R} \mid \mathbf{r}, s_{k}=+1\right)}{\mathrm{P}\left(\hat{s}_{k, R} \mid \mathbf{r}, s_{k}=-1\right)} \\
& =\frac{4 \Re\left[\mathbf{w}_{k}^{H} \mathbf{h}_{k}\right] \cdot \Re\left[\mathbf{w}_{k}^{H}\left(\mathbf{r}-\mathbf{H} \overline{\mathbf{s}}+\bar{s}_{k} \mathbf{h}_{k}\right)\right]}{2 \sigma_{k, R}^{2} / v_{k}^{2}} .
\end{aligned}
$$

\section{SISO Interference Cancellation Using the MBER MUD}

In M-ary PSK systems supporting $K$ users, the transmitted symbol combination may assume $N_{b}=\left(2^{M}\right)^{K}$ possible combinations, here however we limit our discussions to BPSK $(M=1)$ and QPSK $(M=2)$. By defining $x_{k, R}=\operatorname{sgn}\left(s_{k, R}\right) \cdot \hat{s}_{k, R}$, the conditional PDF of $x_{k, R}$ is a Gaussian mixture ${ }^{1}$ given by [11]

$$
\begin{aligned}
\mathrm{P}\left(x_{k, R} ; \mathbf{w}_{k}\right)= & \frac{1}{\sqrt{2 \pi} \sigma_{n} \sqrt{\mathbf{w}_{k}^{H} \mathbf{w}_{k}}} \sum_{q=1}^{N_{b}} \mathrm{P}\left(\mathbf{s}^{(q)}\right) \\
& \cdot \exp \left(-\frac{\left(x_{k, R}-\operatorname{sgn}\left(s_{k, R}^{(q)}\right) \cdot \overline{\hat{s}}_{k, R}^{(q)}\right)^{2}}{2 \sigma_{n}^{2} \mathbf{w}_{k}^{H} \mathbf{w}_{k}}\right),
\end{aligned}
$$

where $\mathrm{P}\left(\mathbf{s}^{(q)}\right)=\prod_{k} \mathrm{P}\left(s_{k}=s_{k}^{(q)}\right)$ is the a priori probability of transmitting the $q$ th possible symbol combination $\mathbf{s}^{(q)}$ of the $K$ users, and $\overline{\hat{s}}_{k, R}^{(q)}$ is the real part of the $k$ th user's estimated symbol, when ignoring the effects of noise

$$
\overline{\hat{s}}_{k, R}^{(q)}=\Re\left[\bar{s}_{k}+v_{k} \mathbf{w}_{k}^{H} \mathbf{H}\left(\mathbf{s}^{(q)}-\overline{\mathbf{s}}\right)\right] .
$$

It can be readily shown that the error probability of the real part is [11]

$$
\begin{aligned}
\mathrm{Pe}_{k, R}\left(\mathbf{w}_{k}\right) & =\mathrm{P}\left(x_{k, R}<0\right)=\int_{-\infty}^{0} \mathrm{P}\left(x_{k, R} ; \mathbf{w}_{k}\right) d x_{k, R} \\
& =\sum_{q=1}^{N_{b}} \mathrm{P}\left(\mathbf{s}^{(q)}\right) \cdot Q\left[\frac{\operatorname{sgn}\left(s_{k, R}^{(q)}\right) \cdot \overline{\hat{s}}_{k, R}^{(q)}}{\sigma_{n} \sqrt{\mathbf{w}_{k}^{H} \mathbf{w}_{k}}}\right] .
\end{aligned}
$$

Similarly, the error probability of the imaginary part is

$$
\mathrm{Pe}_{k, I}\left(\mathbf{w}_{k}\right)=\sum_{q=1}^{N_{b}} \mathrm{P}\left(\mathbf{s}^{(q)}\right) \cdot Q\left[\frac{\operatorname{sgn}\left(s_{k, I}^{(q)}\right) \cdot \overline{\hat{s}}_{k, I}^{(q)}}{\sigma_{n} \sqrt{\mathbf{w}_{k}^{H} \mathbf{w}_{k}}}\right],
$$

where $s_{k, I}^{(q)}$ and $\overline{\hat{s}}_{k, I}^{(q)}$ are the imaginary part of $k$ th user's desired symbol and its estimated version, when the $q$ th $K$-user symbol combination was considered. Hence the BER of the beamformer is

$$
\mathrm{Pe}_{k}= \begin{cases}\mathrm{Pe}_{k, R}, & \text { BPSK } \\ \frac{1}{2}\left(\mathrm{Pe}_{k, R}+\mathrm{Pe}_{k, I}\right), & \text { QPSK } .\end{cases}
$$

The MBER beamforming solution is then defined as [11]

$$
\mathbf{w}_{k, \text { mber }}=\arg \min _{\mathbf{w}} \mathrm{Pe}_{k}\left(\mathbf{w}_{k}\right) \text {. }
$$

\footnotetext{
${ }^{1}$ A Gaussian mixture is constituted by the weighted sum of Gaussian densities, where the weights are all positive and sum to unity.
}

This optimization problem can be solved using the simplified conjugate gradient algorithm, which is detailed in [11]. The gradients of both the inphase and quadrature-phase bit error probabilities are

$$
\begin{aligned}
\nabla \mathrm{Pe}_{k, R}\left(\overline{\mathbf{w}}_{k}\right)= & \frac{1}{\sqrt{2 \pi} \sigma_{n}} \sum_{q=1}^{N_{b}} \mathrm{P}\left(\mathbf{s}^{(q)}\right) \cdot \exp \left(-\frac{\left(\overline{\hat{s}}_{k, R}^{(q)}\right)^{2}}{2 \sigma_{n}^{2}}\right) \\
& \cdot \operatorname{sgn}\left(s_{k, R}^{(q)}\right)\left(\overline{\mathbf{w}}_{k} \overline{\hat{s}}_{k, R}^{(q)}-v_{k} \mathbf{H}\left(\mathbf{s}^{(q)}-\overline{\mathbf{s}}\right)\right)
\end{aligned}
$$

and

$$
\begin{aligned}
\nabla \mathrm{Pe}_{k, I}\left(\overline{\mathbf{w}}_{k}\right)= & \frac{1}{\sqrt{2 \pi} \sigma_{n}} \sum_{q=1}^{N_{b}} \mathrm{P}\left(\mathbf{s}^{(q)}\right) \cdot \exp \left(-\frac{\left(\overline{\hat{s}}_{k, I}^{(q)}\right)^{2}}{2 \sigma_{n}^{2}}\right) \\
& \cdot \operatorname{sgn}\left(s_{k, I}^{(q)}\right)\left(\overline{\mathbf{w}}_{k} \overline{\hat{s}}_{k, I}^{(q)}+j v_{k} \mathbf{H}\left(\mathbf{s}^{(q)}-\overline{\mathbf{s}}\right)\right),
\end{aligned}
$$

where $\overline{\mathbf{w}}_{k}$ is the normalized i.e. unity-norm version of the vector $\mathbf{w}_{k}$.

In the MBER design, both the real part and imaginary part of the estimated symbols are non-Gaussian. Hence we cannot use the Gaussian approximation for calculating the output extrinsic LLRs of the MBER multiuser detector. The exact expression of the extrinsic information delivered by the MUD is [6]

$$
\ln \frac{\sum_{\forall \mathbf{s}(q): b_{k}^{(q)}(i)=0}^{L_{e, b_{k}(i)}} \mathrm{P}\left(\hat{s}_{k, i} \mid \mathbf{s}^{(q)}\right) \prod_{\forall k^{\prime}, i^{\prime}:\left(k^{\prime}, i^{\prime}\right) \neq(k, i)} \mathrm{P}\left(b_{k^{\prime}}\left(i^{\prime}\right)^{(q)}\right)}{\sum_{\forall \mathbf{s}(q): b_{k}^{(q)}(i)=1} \mathrm{P}\left(\hat{s}_{k, i} \mid \mathbf{s}^{(q)}\right) \prod_{\forall k^{\prime}, i^{\prime}:\left(k^{\prime}, i^{\prime}\right) \neq(k, i)} \mathrm{P}\left(b_{k^{\prime}}\left(i^{\prime}\right)^{(q)}\right)},
$$

where we have

$$
\begin{aligned}
& \mathrm{P}\left(\hat{s}_{k, 1} \mid \mathbf{s}^{(q)}\right)=\frac{1}{\sqrt{2 \pi} \sigma_{n}} \exp \left(-\frac{\Re^{2}\left[\overline{\mathbf{w}}_{k}^{H}\left(\mathbf{r}-\mathbf{H s}^{(q)}\right)\right]}{2 \sigma_{n}^{2}}\right), \\
& \mathrm{P}\left(\hat{s}_{k, 2} \mid \mathbf{s}^{(q)}\right)=\frac{1}{\sqrt{2 \pi} \sigma_{n}} \exp \left(-\frac{\Im^{2}\left[\overline{\mathbf{w}}_{k}^{H}\left(\mathbf{r}-\mathbf{H} \mathbf{s}^{(q)}\right)\right]}{2 \sigma_{n}^{2}}\right),
\end{aligned}
$$

which represents the conditional probability of the real and imaginary part of the $k$ th user's estimated symbol, when transmitting the $q$ th combination $\mathbf{s}^{(q)}$. Furthermore [3]

$$
\mathrm{P}\left(b_{k^{\prime}}\left(i^{\prime}\right)^{(q)}\right)=\frac{1+\operatorname{sgn}\left(b_{k^{\prime}}\left(i^{\prime}\right)^{(q)}\right) \tanh \left(\frac{L_{p r, b_{k^{\prime}\left(i^{\prime}\right)}}}{2}\right)}{2}
$$

is the probability of the $k^{\prime}$ th user's $i^{\prime}$ th bit in case of the $q$ th $K$-user symbol combination using the a priori information.

\section{Simulation Results}

In this section, we present our simulation results, in order to illustrate the performance of the iterative beamforming receiver. The BER performance of the CMMSE, RMMSE and MBER algorithms is plotted in Fig. 3 and Fig. 4. The system employs a two-element antenna array and supports up to $K=6$ users. All users employ BPSK modulation and have the same transmit power and channel coefficent of $A_{k}=1.0+j 0.0$ (for $1 \leq k \leq K$ ). The locations of users with respect to the antenna array are listed in Table I. All users employ the same rate $1 / 2$ and constraint length 3 RSC code using the generators 13,6 in octal notation. Each user employs a different interleaver generated randomly. The interleaver length of each user is 1000 bits.

TABLE I

ARRIVAL ANGLES OF THE USERS' SIGNALS

\begin{tabular}{c|cccccc} 
user $k$ & 1 & 2 & 3 & 4 & 5 & 6 \\
\hline$\theta_{k}$ & $15^{\circ}$ & $-4^{\circ}$ & $36^{\circ}$ & $-24^{\circ}$ & $68^{\circ}$ & $-48^{\circ}$
\end{tabular}




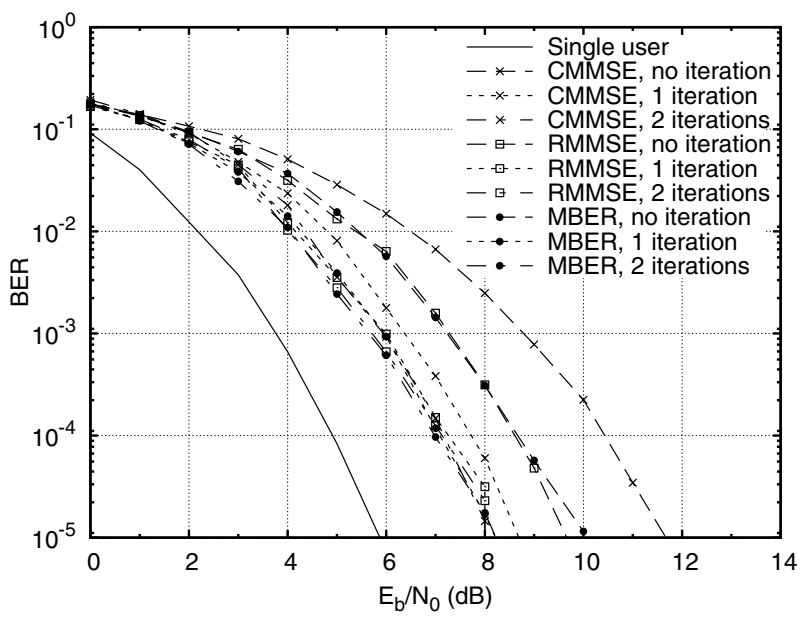

Fig. 3. BER comparison of the CMMSE, RMMSE and MBER iterative beamforming receivers for the BPSK system supporting $K=2$ users employing a two-element array

In Fig. 3, only the first two users are activated and the singleuser performance is also included as a reference. It can be seen that after two iterations, the CMMSE, RMMSE and MBER algorithms exhibit an iteration gain of $3.5 \mathrm{~dB}, 1.3 \mathrm{~dB}$ and $1.9 \mathrm{~dB}$, respectively, at a BER of $10^{-5}$. At the same time, the MBER receiver's performance is almost the same as that of the CMMSE and RMMSE solutions. The achievable performance gain is modest in this two-user scenario, since the performance is already relatively close to the single-user bound and no further iteration gains are attained for more iterations. This limited iteration gain is the consequence of the limited extrinsic information associated with a simple 2-user scenario.

To elaborate a little further, Fig. 4 shows the attainable performance, when the number of users is increased to six. It can be seen that the performance of both the MBER and the RMMSE beamforming receivers has significantly improved after six iterations. However, the CMMSE solution is no longer able to provide the desired user seperation, resulting in a high BER floor. It is also seen that in this 'overloaded' system supporting three times the number of users in comparison to the number of antennas, the MBER algorithm has the lowest residual BER. After six iterations, the MBER receiver has $1.2 \mathrm{~dB}$ gain over the RMMSE solution at a BER of $10^{-4}$.

It should also be mentioned that in all simulations discussed above, the angular locations of the users were selected to render the user's BERs similar. If some of the users exhibit a high BER, this would impose an error propagation phenomenon, potentially limiting the achievable multiuser performance.

\section{CONCLUSION}

In this paper, we introduced a new iterative MBER soft interference cancellation beamforming receiver, which directly minimizes the BER instead of the MSE. The RMMSE algorithm designed for BPSK was also considered, which minimizes the MSE between the real-valued desired signal and the real part of the complex-valued beamformer output. Our simulations have shown that the MBER solution outperforms both the conventional CMMSE and the RMMSE iterative receivers. Our future research will consider similar wideband scenarios and more sophisticated channel codecs.

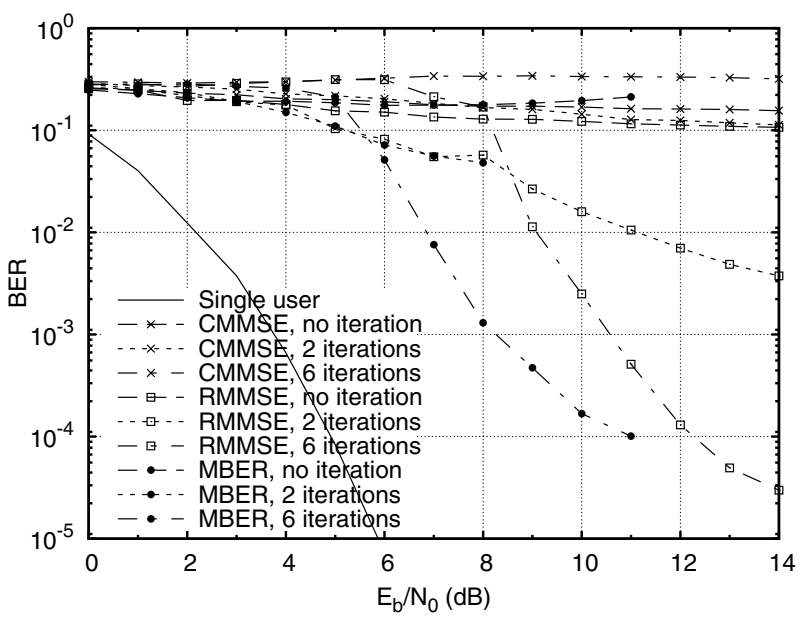

Fig. 4. BER comparison of the CMMSE, RMMSE and MBER iterative beamforming receivers for the BPSK system supporting $K=6$ users employing a two-element array

\section{REFERENCES}

[1] J. S. Blogh and L. Hanzo, Third Generation Systems and Intelligent Wireless Networking - Smart Anttenas and Adaptive Modulation. Chichester. John Wiley and IEEE Press, 2002

[2] L. Hanzo, M. Münster, B. J. Choi, and T. Keller, OFDM and MC-CDMA for Broadband Multi-user Communications, WLANs and Broadcasting. John Wiley and IEEE Press, 2003

[3] C. Berrou, A. Glavieux, and P. Thitimajshima, "Near Shannon limit error correcting coding and decoding: Turbo codes," in Proc. IEEE Int. Conf. Commun., Vol.2, Geneva, Switzerland, pp.1064-1070, May 1993

[4] L. Hanzo, T. H. Liew, and B. L. Yeap, Turbo Coding, Turbo Equalisation and Space-Time Coding for Transmission over Fading Channels. John Wiley and IEEE Press, 2002

[5] L. Hanzo, C. H. Wong, and M. S. Yee, Adaptive Wireless Transceivers: Turbo-Coded, Turbo-Equalised and Space-Time Coded TDMA, CDMA and OFDM Systems. John Wiley and IEEE Press, 2002

[6] L. Hanzo, L. L. Yang, E. L. Kuan, and K. Yen, Single-and Multi-Carrier DS-CDMA: Multi-User Detection, Space-Time Spreading, Synchronisation, Standards and Networking. John Wiley and IEEE Press, 2003

[7] X. Wang and H. V. Poor, "Iterative (Turbo) soft interference cancellation and decoding for coded CDMA," IEEE Trans. Commun., Vol.47, No.7, pp.1046-1060, July 1999

[8] M. Tüchler, A. C. Singer, and R. Koetter, "Minimum mean squared error equalization using a priori information," IEEE Trans. Signal Processing, Vol.50, No.3, pp.673-6820, March 2002

[9] A. Tarable, G. Montorsi, and S. Benedetto, "A linear front end for iterative soft interference cancellation and decoding in coded CDMA," IEEE Trans. Wireless Communications, Vol.4, No.2, pp.507-518, March 2005

[10] R. Schober, W. H. Gerstacker, and L. Lampe, "On suboptimum receivers for DS-CDMA with BPSK modulation," Signal Processing, Vol.85, No.6, pp.1149-1163, June 2005

[11] S. Chen, N. N. Ahmad, and L. Hanzo, "Adaptive minimum bit error rate beamforming," IEEE Trans. Wireless Communications, Vol.4, No.2, pp.341-348, March 2005

[12] A. Wolfgang, N. N. Ahmad, S. Chen, and L. Hanzo, "Genetic algorithm assisted minimum bit error rate beamforming," in Proc. VTC2004Spring, pp.142-146, Milan, Italy

[13] M. Y. Alias, A. K. Samingan, S. Chen, and L. Hanzo, "Multiple antenna aided OFDM employing minimum bit error rate multiuser detection," IEE Electronics Letter, Vol.39, No.24, pp.1769-1770, November 2003

[14] H. V. Poor and S. Verdú, "Probability of error in MMSE multiuser detection," IEEE Trans. Inf. Theory, Vol.IT-43, pp.858-871, May 1997 